\title{
Finite Larmor Radius and Hall Effects on Thermal Instability of a Plasma in Porous Medium
}

\author{
R. C. Sharma \\ Department of Mathematics, Himachal Pradesh University, Shimla-171 005, India \\ V.K. Bhardwaj \\ Department of Mathematics, St. Bedes College, Shimla, India
}

Z. Naturforsch. 49a, 547-551 (1994); received December 31, 1993

\begin{abstract}
The thermal instability of a plasma in a porous medium in the presence of a finite Larmor radius (FLR) and Hall effects is considered. Oscillatory modes due to the presence of a magnetic field (and hence the presence of FLR and Hall effects) are introduced. For stationary convection, the FLR may have a stabilizing or destabilizing effect, but a completely stabilizing one for a certain wave-number range. Similarly, the Hall currents may have a stabilizing or destabilizing effect but a completely stabilizing one for the same wave-number range under certain condition, whereas the medium permeability always has a destabilizing effect for stationary convection.
\end{abstract}

\section{Introduction}

The effects of finiteness of the ion Larmor radius, showing up in the form of a magnetic viscosity in the fluid equations, have been studied by Rosenbluth et al. [1], Roberts and Taylor [2], Vandakurov [3] and Jukes [4]. Melchior and Popowich [5] have considered the finite Larmor radius (FLR) effect on the KelvinHelmholtz instability of a plasma, while that on the Rayleigh-Taylor instability has been studied by Singh and Hans [6]. The theory of thermal instability of a fluid layer heated from below under varying assumptions of hydromagnetics has been summarized by Chandrasekhar [7]. The effect of FLR on the thermal instability of a plasma has been studied by Sharma [8] in the presence of a vertical magnetic field and by Sharma and Prakash [9] in the presence of a horizontal magnetic field. Gupta [10] has studied the thermal instability of a conducting fluid in the presence of Hall current. The medium has been considered to be nonporous in all the above studies.

The problem of thermal convection in fluids in a porous medium is of importance in geophysics, soil sciences, ground water hydrology and astrophysics. The development of geothermal power resources has increased general interest in the properties of convec-

Reprint requests to Prof. Dr. R. C. Sharma, Department of Mathematics, Himachal Pradesh University, Shimla-171 005, Indien. tion in porous media. Generally it is accepted that comets consist of a dusty 'snowball' of a mixture of frozen gases which in the process of their journey changes from solid to gas and vice-versa. The physical properties of comets, meteorites and interplanetary dust strongly suggest the importance of porosity in the astrophysical context (McDonnel [11]). The effect of a magnetic field on the stability of such a flow is of interest in geophysics, particularly in the study of the Earth's core where the Earth's mantle, which consists of a conducting fluid, behaves like a porous medium which can become convectively unstable as a result of differential diffusion. The other application of the results of flow through a porous medium in the presence of a magnetic field is in the study of the stability of a convective flow in the geothermal region.

Keeping in mind the importance of FLR and Hall currents in several situations of geophysics and astrophysics, the present paper deals with the thermal instability of a plasma in a porous medium in the presence of FLR and Hall current effects.

\section{Perturbation Equations}

Consider an infinite horizontal layer of viscous, heat conducting and finite electrically conducting fluid of thickness $d$, in porous medium, in which a uniform temperature gradient $\beta(=|d T / \mathrm{d} z|)$ is maintained. Consider the cartesian coordinates $(x, y, z)$ 
with origin on the lower boundary $z=0$ and the $z$-axis perpendicular to it along the vertical. The fluid is acted on by a horizontal magnetic field $\boldsymbol{H}(H, 0,0)$ and gravity force $\boldsymbol{g}(0,0,-g)$.

Let $\boldsymbol{q}(u, v, w), \boldsymbol{h}\left(h_{x}, h_{y}, h_{z}\right), \delta \varrho$ and $\theta$ denote respectively the perturbations in fluid (filter) velocity $(0,0,0)$, magnetic field $\boldsymbol{H}$, density $\varrho$ and temperature $T$. Then the linearized hydromagnetic perturbation equations relevant to the problem are

$$
\begin{aligned}
\frac{1}{\varepsilon} \frac{\partial \boldsymbol{q}}{\partial t}= & -\frac{1}{\varrho_{0}} \nabla \mathbf{P}-\frac{1}{\varrho_{0}} \nabla \delta p-\frac{v}{k_{1}} \boldsymbol{q} \\
& +\frac{1}{4 \pi \varrho_{0}}(\nabla \times \boldsymbol{h}) \times \boldsymbol{H}-\boldsymbol{g} \alpha \theta, \\
\nabla \cdot \boldsymbol{q}= & 0, \\
\varepsilon \frac{\partial \boldsymbol{h}}{\partial t}= & \nabla \times(\boldsymbol{q} \times \boldsymbol{H})+\varepsilon \eta \nabla^{2} \boldsymbol{h} \\
& -\left(\frac{\varepsilon}{4 \pi N e}\right) \nabla \times[(\nabla \times \boldsymbol{h}) \times \boldsymbol{H}], \\
\nabla \cdot \boldsymbol{h}= & 0, \\
E \frac{\partial \theta}{\partial t}= & \beta w+\chi \nabla^{2} \theta,
\end{aligned}
$$

where

Here

$$
E=\varepsilon+(1-\varepsilon) \frac{\varrho_{\mathrm{s}} c_{\mathrm{s}}}{\varrho_{0} c_{v}} .
$$

$\mathbf{P}, \varrho_{0}, \mu, v\left(=\mu / \varrho_{0}\right), K, \chi\left(=\frac{K}{\varrho_{0} c_{v}}\right), \eta, \alpha, N$ and $e$

stand for stress tensor taking into account FLR effect, density at reference level $z=0$, viscosity, kinematic viscosity, thermal conductivity, thermal diffusivity, electrical resistivity, coefficient of volume expansion, electron number density and charge of an electron respectively. $\varepsilon, k_{1}$ denote the medium porosity, medium permeability while $\varrho_{0}, c_{v} ; \varrho_{s}, c_{\mathrm{s}}$ stand for density and specific heat of fluid and solid (porous matrix) material respectively. In writing (1), use has been made of the equation of state

$$
\varrho=\varrho_{0}\left[1-\alpha\left(T-T_{0}\right)\right],
$$

where the suffix zero refers to values at the reference level $z=0$ and so the change in density $\delta \varrho$ caused by the perturbation $\theta$ in temperature is given by

$$
\delta \varrho=-\alpha \varrho_{0} \theta .
$$

For the horizontal magnetic field $\boldsymbol{H}(H, 0,0)$, the stress tensor $\mathbf{P}$, taking into account the finite ion gyra- tion (Vandakurov [3]), has the components

$P_{x x}=0, P_{x y}=P_{y x}=-2 \varrho_{0} v_{0}\left(\frac{\partial w}{\partial x}+\frac{\partial u}{\partial z}\right)$,

$P_{x z}=P_{z x}=2 \varrho_{0} v_{0}\left(\frac{\partial u}{\partial y}+\frac{\partial v}{\partial x}\right)$,

$P_{y y}=-\varrho_{0} v_{0}\left(\frac{\partial v}{\partial z}+\frac{\partial w}{\partial y}\right)$,

$P_{y z}=P_{z y}=\varrho_{0} v_{0}\left(\frac{\partial v}{\partial y}-\frac{\partial w}{\partial z}\right), P_{z z}=\varrho_{0} v_{0}\left(\frac{\partial v}{\partial z}+\frac{\partial w}{\partial y}\right)$.

Here $\varrho_{0} v_{0}=\frac{N T}{4 \omega_{\mathrm{H}}}$, where $N, T$, and $\omega_{\mathrm{H}}$ denote respectively the number density, the ion temperature and the ion gyration frequency.

\section{Dispersion Relation}

Here we analyze the disturbances into normal modes and assume the perturbation quantities are of the form

$$
\begin{aligned}
{\left[w, \theta, h_{z}, \zeta, \xi\right]=} & {[W(z), \Theta(z), K(z), Z(z), X(z)] } \\
& \cdot \exp \left(i k_{x} x+i k_{y} y+n t\right),
\end{aligned}
$$

where $k_{x}, k_{y}$ are the wave numbers in the $x$ - and $y$-directions respectively, $k=\left(k_{x}^{2}+k_{y}^{2}\right)^{1 / 2}$ is the resultant wave number and $n$ is, in general, a complex constant.

Expressing the coordinates $x, y, z$ in the new unit of length $d$ and letting $a=k d, \sigma=\frac{n d^{2}}{v}, p_{1}=\frac{v}{x}, p_{2}=\frac{v}{\eta}$ and $D=\frac{\mathrm{d}}{\mathrm{d} z} ;(1)-(5)$ using (8) and expression (9), give

$$
\begin{aligned}
\left(\frac{\sigma}{\varepsilon}+\frac{1}{P_{l}}\right)\left(D^{2}-a^{2}\right) W+\frac{g \alpha d^{2}}{v} a^{2} \Theta \\
-\frac{i k_{x} H d^{2}}{4 \pi \varrho_{0} v}\left(D^{2}-a^{2}\right) K \\
-\left(\frac{i k_{x} v_{0} d^{2}}{v}\right)\left(D^{2}-a^{2}+3 \frac{k_{x}^{2}}{k^{2}} a^{2}\right) Z=0,
\end{aligned}
$$

$$
\begin{aligned}
\left(\frac{\sigma}{\varepsilon}+\frac{1}{P_{l}}\right) Z= & -\left(\frac{i k_{x} v_{0}}{v}\right)\left(D^{2}-a^{2}+3 \frac{k_{x}^{2}}{k^{2}} a^{2}\right) W \\
& +\frac{i k_{x} H d^{2}}{4 \pi \varrho_{0} v} X
\end{aligned}
$$

$\left(D^{2}-a^{2}-p_{2} \sigma\right) K=-\frac{i k_{x} H d^{2}}{\varepsilon \eta} W+\frac{i k_{x} H d^{2}}{4 \pi N e \eta} X$, 


$$
\begin{aligned}
\left(D^{2}-a^{2}-p_{2} \sigma\right) X= & -\frac{i k_{x} H d^{2}}{\varepsilon \eta} Z \\
& -\frac{i k_{x} H}{4 \pi N e \eta}\left(D^{2}-a^{2}\right) K,
\end{aligned}
$$

$\left(D^{2}-a^{2}-E p_{1} \sigma\right) \Theta=-\left(\frac{\beta d^{2}}{\varkappa}\right) W$.

Eliminating $\Theta, K, X$, and $Z$ between (10)-(14), we obtain

$$
\begin{aligned}
{\left[\left(\frac{\sigma}{\varepsilon}+\frac{1}{P_{l}}\right)\left(D^{2}-a^{2}-p_{2} \sigma\right)^{2}-Q k_{x}^{2} d^{2}\left(D^{2}-a^{2}-p_{2} \sigma\right)\right.} \\
\left.-M k_{x}^{2} d^{2}\left(\frac{\sigma}{\varepsilon}+\frac{1}{P_{l}}\right)\left(D^{2}-a^{2}\right)\right] \\
\cdot\left[\left(\frac{\sigma}{\varepsilon}+\frac{1}{P_{l}}\right)\left(D^{2}-a^{2}\right)\left(D^{2}-a^{2}-E p_{1} \sigma\right)-R a^{2}\right] \\
\cdot W-Q k_{x}^{2} d^{2}\left(D^{2}-a^{2}-E p_{1} \sigma\right)\left(D^{2}-a^{2}\right) \\
\cdot\left[\left\{\left(\frac{\sigma}{\varepsilon}+\frac{1}{P_{l}}\right)\left(D^{2}-a^{2}-p_{2} \sigma\right)-Q k_{x}^{2} d^{2}\right\}\right. \\
\left.-2 M^{1 / 2} N^{1 / 2} k_{x}^{2} d^{2}\left(D^{2}-a^{2}+3 \frac{k_{x}^{2}}{k^{2}} a^{2}\right)\right] W \\
-N k_{x}^{2} d^{2}\left(D^{2}-a^{2}-E p_{1} \sigma\right)\left(D^{2}-a^{2}+3 \frac{k_{x}^{2}}{k^{2}} a^{2}\right)^{2} \\
\cdot\left[\left(D^{2}-a^{2}-p_{2} \sigma\right)^{2}-M k_{x}^{2} d^{2}\left(D^{2}-a^{2}\right)\right] W=0,
\end{aligned}
$$

where

$$
R=\frac{g \alpha \beta d^{4}}{v \varkappa}
$$

is the Rayleigh number,

$$
Q=\frac{H^{2} d^{2}}{4 \pi \varrho_{0} v \eta \varepsilon}
$$

is the modified Chandrasekhar number,

$$
M=\left(\frac{\mathrm{H}}{4 \pi N e \eta}\right)^{2}
$$

is a nondimensional number accounting for Hall current,

$$
N=\left(\frac{v_{0}}{v}\right)^{2}
$$

is a nondimensional number accounting for FLR effects and $P_{l}=\frac{k_{1}}{\varepsilon d^{2}}$.

Consider the case of plasma layer with two free boundaries and the adjoining medium to be noncon- ducting. The boundaries are assumed to be perfect conductors of heat. The case of two free boundaries is the most appropriate for stellar atmospheres (Spiegel [12]). The boundary conditions appropriate for the problem are

$$
\begin{aligned}
& W=\Theta=D^{2} W=D Z=0 \quad \text { at } z=0 \text { and } 1 \\
& X=0 \text { and } h_{x}, h_{y}, h_{z} \text { are continuous. }
\end{aligned}
$$

Using the boundary conditions (16), it can be shown with the help of (10)-(14) that all the even order derivatives of $W$ vanish at the boundaries and hence the proper solution of (15) characterizing the lowest mode is

$$
W=W_{0} \sin \pi z,
$$

where $W_{0}$ is a constant. Substituting (17) in (15) and letting

$a^{2}=\pi^{2} x, R_{1}=\frac{R}{\pi^{4}}, Q_{1}=\frac{Q}{\pi^{2}}, k_{x}=k \cos \theta, i \sigma_{1}=\frac{\sigma}{\pi^{2}}$ and

$$
P=\pi^{2} P_{l},
$$

we obtain the dispersion relation

$$
\begin{aligned}
R_{1}= & \left(\frac{i \sigma_{1}}{\varepsilon}+\frac{1}{P}\right)\left(\frac{1+x}{x}\right)\left(1+x+i E p_{1} \sigma_{1}\right) \\
& +\left[Q_{1} \cos ^{2} \theta(1+x)\left(1+x+i E p_{1} \sigma_{1}\right)\right. \\
& \cdot\left\{\left(\frac{i \sigma_{1}}{\varepsilon}+\frac{1}{P}\right)\left(1+x+i p_{2} \sigma_{1}\right)+Q_{1} x \cos ^{2} \theta\right\} \\
& +2 M^{1 / 2} N^{1 / 2} Q_{1}\left(\cos ^{4} \theta\right) x(1+x)\left(1+x+i E p_{1} \sigma_{1}\right) \\
& \cdot\left(1+x-3 x \cos ^{2} \theta\right)+N \cos ^{2} \theta\left(1+x+i E p_{1} \sigma_{1}\right) \\
& \cdot\left(1+x-3 x \cos ^{2} \theta\right)^{2} \\
& \left.\cdot\left\{\left(1+x+i p_{2} \sigma_{1}\right)^{2}+M x(1+x) \cos ^{2} \theta\right\}\right] \\
& \cdot\left[\left(\frac{i \sigma_{1}}{\varepsilon}+\frac{1}{P}\right)\left(1+x+i p_{2} \sigma_{1}\right)^{2}\right. \\
& +Q_{1} x \cos ^{2} \theta\left(1+x+i p_{2} \sigma_{1}\right) \\
& \left.+M x(1+x)\left(\frac{i \sigma_{1}}{\varepsilon}+\frac{1}{P}\right) \cos ^{2} \theta\right]^{-1} .
\end{aligned}
$$

\section{The Stationary Convection}

When the instability sets in as stationary convection, the marginal state will be characterized by $\sigma=0$. 
Putting $\sigma=0$, the dispersion relation (18) reduces to

$$
\begin{aligned}
R_{1} & =\frac{(1+x)^{2}}{x P} \\
& +\left[Q_{1}(1+x) \cos ^{2} \theta\left(\frac{1+x}{P}+Q_{1} x \cos ^{2} \theta\right)\right. \\
& +2 M^{1 / 2} N^{1 / 2} Q_{1}\left(\cos ^{4} \theta\right) x\left(1+x-3 x \cos ^{2} \theta\right)(1+x) \\
& +N \cos ^{2} \theta(1+x) \\
& \left.\cdot\left(1+x-3 x \cos ^{2} \theta\right)^{2}\left(1+x+M x \cos ^{2} \theta\right)\right] \\
& \cdot\left[\frac{1+x}{P}+Q_{1} x \cos ^{2} \theta+\frac{M x \cos ^{2} \theta}{P}\right]^{-1}
\end{aligned}
$$

which expresses the modified Rayleigh number $R_{1}$ as a function of the dimensionless wave number $x$ and the parameters $Q_{1}, M, N$ and $P$. From (19), it follows that

$$
\begin{aligned}
\frac{\mathrm{d} R_{1}}{\mathrm{~d} N}= & {\left[(1+x)\left(1+x-3 x \cos ^{2} \theta\right)^{2}\right.} \\
& \cdot\left(1+x+M x \cos ^{2} \theta\right) \cos ^{2} \theta \\
& \left.+\left(\frac{M}{N}\right)^{1 / 2} Q_{1} x(1+x)\left(1+x-3 x \cos ^{2} \theta\right) \cos ^{4} \theta\right] \\
& \cdot\left[\frac{1+x}{P}+Q_{1} x \cos ^{2} \theta+\frac{M x \cos ^{2} \theta}{P}\right]^{-1},
\end{aligned}
$$

which is positive for $(1+x)>3 x \cos ^{2} \theta$ i.e. for the wave number range satisfying

$$
\cos \theta<\left\{\frac{(1+x)}{3 x}\right\}^{1 / 2} .
$$

This shows that FLR has a stabilizing effect for the wave number range (21). In the absence of Hall currents $(M \rightarrow 0)$, FLR always has a stabilizing effect. But in the presence of FLR and Hall effects on the thermal instability in porous medium, the FLR may have stabilizing or destabilizing effects but completely stabilizes the wave number range (21).

Equation (19) also yields

$$
\begin{aligned}
\frac{\mathrm{d} R_{1}}{\mathrm{~d} M}= & {\left[N x(1+x)\left(1+x-3 x \cos ^{2} \theta\right)^{2} \cos ^{4} \theta\right.} \\
& \cdot\left(\frac{1+x}{P}+Q_{1} x \cos ^{2} \theta\right) \\
& +\left(\frac{N}{M}\right)^{1 / 2} \frac{Q_{1}}{P} x(1+x)^{2}\left(1+x-3 x \cos ^{2} \theta\right) \cos ^{4} \theta
\end{aligned}
$$

$$
\begin{aligned}
& +Q_{1} x^{2} \cos ^{6} \theta\left(1+x-3 x \cos ^{2} \theta\right) \\
& \left.\cdot \frac{1}{P}\left(\frac{N}{M}\right)^{1 / 2}\left(P Q_{1}-M\right)\right] \\
& \cdot\left[\frac{1}{P}\left(1+x+M x \cos ^{2} \theta\right)+Q_{1} x \cos ^{2} \theta\right]^{-2},
\end{aligned}
$$

which is positive if $P Q_{1}>M$ and $(1+x)>3 x \cos ^{2} \theta$ i.e. if

$\frac{k_{1}}{\varrho_{0} v}>\frac{\varepsilon^{2}}{4 \pi^{3} \eta N^{2} e^{2}}$ and $\cos \theta<\left(\frac{(1+x)}{3 x}\right)^{1 / 2}$.

In the presence of FLR and Hall effects on the thermal instability in porous medium, the Hall currents may have stabilizing or destabilizing effect but completely stabilizes the wave number range (21) if

$$
\frac{k_{1}}{\varrho_{0} v}>\frac{\varepsilon^{2}}{4 \pi^{3} \eta N^{2} e^{2}} .
$$

It is evident from (19) that

$$
\begin{aligned}
\frac{\mathrm{d} R_{1}}{\mathrm{~d} P}= & -\left[(1+x)^{4}+2 x(1+x)^{3}\left(P Q_{1}+M\right) \cos ^{2} \theta\right. \\
& +x^{2} M(1+x)^{2}\left(M+2 P Q_{1}\right) \cos ^{4} \theta \\
& \left.+2 P^{2} Q_{1}^{2} x^{2}(1+x)^{2} \cos ^{4} \theta\right] \\
& \cdot\left[P^{2} x\left\{1+x+x \cos ^{2} \theta\left(P Q_{1}+M\right)\right\}\right]^{-2},
\end{aligned}
$$

which means that medium permeability has a destabilizing effect.

\section{Stability of the System and Oscillatory Modes}

Multiplying equation (10) by $W^{*}$, the complex conjugate of $W$, integrating over the range of $z$ and making use of (11)-(14), we obtain

$$
\begin{aligned}
\left(\frac{\sigma}{\varepsilon}+\frac{1}{P_{l}}\right) I_{1}+\frac{\varepsilon \eta}{4 \pi \varrho_{0} v}\left(I_{4}+p_{2} \sigma^{*} I_{5}\right) \\
+\frac{\varepsilon \eta d^{2}}{4 \pi \varrho_{0} v}\left(I_{6}+p_{2} \sigma I_{7}\right) \\
+d^{2}\left(\frac{\sigma^{*}}{\varepsilon}+\frac{1}{P_{l}}\right) I_{8}=\frac{g \alpha \varkappa a^{2}}{v \beta}\left(I_{2}+E p_{1} \sigma^{*} I_{3}\right)
\end{aligned}
$$

where

$$
\begin{aligned}
& I_{1}=\int_{0}^{1}\left(|D W|^{2}+a^{2}|W|^{2}\right) \mathrm{d} z, \\
& I_{2}=\int_{0}^{1}\left(|D \Theta|^{2}+a^{2}|\Theta|^{2}\right) \mathrm{d} z,
\end{aligned}
$$




$$
\begin{aligned}
& I_{3}=\int_{0}^{1}|\Theta|^{2} \mathrm{~d} z, \\
& I_{4}=\int_{0}^{1}\left(\left|D^{2} K\right|^{2}+2 a^{2}|D K|^{2}+a^{4}|K|^{2}\right) \mathrm{d} z, \\
& I_{5}=\int_{0}^{1}\left(|D K|^{2}+a^{2}|K|^{2}\right) \mathrm{d} z, \\
& I_{6}=\int_{0}^{1}\left(|D X|^{2}+a^{2}|X|^{2}\right) \mathrm{d} z, \\
& I_{7}=\int_{0}^{1}|X|^{2} \mathrm{~d} z, \\
& I_{8}=\int_{0}^{1}|Z|^{2} \mathrm{~d} z,
\end{aligned}
$$$$
=-\left[\frac{I_{1}}{P_{l}}+\frac{\varepsilon \eta}{4 \pi \varrho_{0} v}\left(I_{4}+d^{2} I_{6}\right)\right.
$$$$
\left.+\frac{d^{2}}{P_{l}} I_{8}-\frac{g \alpha \varkappa a^{2}}{v \beta} I_{2}\right],
$$

and

$$
\begin{gathered}
\sigma_{i}\left[\frac{I_{1}}{\varepsilon}+\frac{\varepsilon \eta p_{2}}{4 \pi \varrho_{0} v}\left(d^{2} I_{7}-I_{5}\right)-\frac{d^{2}}{\varepsilon} I_{8}\right. \\
\left.+\frac{g \propto \varkappa a^{2}}{v \beta} E p_{1} I_{3}\right]=0 .
\end{gathered}
$$

It is evident from (27) that $\sigma_{i}$ may be zero or non-zero. Thus the modes may be oscillatory or non-oscillatory. The oscillatory modes are introduced due to the presence of magnetic field (and hence the presence of FLR and Hall effects). In the absence of magnetic field, the oscillatory modes are not allowed but in the presence of magnetic field, Hall currents and FLR effects, oscillatory modes come into play. Equation (26) simply tells that $\sigma_{\gamma}$ may be positive or negative meaning thereby that the system may be stable or unstable.

[8] R. C. Sharma, Nuovo Cim. 20 B, 303 (1974).

[1] M. N. Rosenbluth, N. Krall, and N. Rostoker, Nucl. Fusion Suppl. 1, 143 (1962).

[2] K. V. Roberts and J. B. Taylor, Phys. Rev. Letters 8, 197 (1962).

[3] Ju. V. Vandakurov, Prik. Mat. Mech. 28, 69 (1964).

[4] J. D. Jukes, Phys. Fluids 7, 52 (1964).

[5] H. Melchior and M. Popowich, Phys. Fluids 11, 581 (1968).

[6] H. K. Hans, Nucl. Fusion 8, 89 (1968).

[7] S. Chandrasekhar, Hydrodynamic and Hydromagnetic Stability, Dover Publication, New York 1981.

[9] R. C. Sharma and K. Prakash, Z. Naturforsch. 30a, 461 (1975).

[10] A. S. Gupta, Rev. Roumaine Math. Pures Appl. 12, 665 (1967).

[11] J. A. M. McDonnel, Cosmic Dust, John Wiley and Sons, Toronto 1978, p. 330.

[12] E. A. Spiegel, Astrophys. J. 141, 1068 (1965). 